\title{
Nanoscale
}

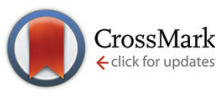

Cite this: Nanoscale, 2014, 6, 14523

\section{Ostwald-ripening and particle size focussing of sub-10 nm NaYF 4 upconversion nanocrystals $\uparrow$}

\author{
Thorben Rinkel, Jörg Nordmann, Athira Naduviledathu Raj and Markus Haase*
}

Received 9th July 2014 Accepted 29th September 2014 DOI: $10.1039 / c 4 n r 03833 a$ www.rsc.org/nanoscale

\begin{abstract}
We have studied the growth behaviour of sub-10 nm NaYF 4 upconversion nanocrystals of the hexagonal $\beta$-phase and the cubic $\alpha$-phase. Ostwald-ripening of such particles in oleic acid/octadecene solvent results in broadening of the particle size distribution if the colloid contains particles of one crystal phase only. Narrow size distributions are formed only if $\beta$-phase particles grow in the presence of an excess of $\alpha$-phase particles. Such binary mixtures of $\alpha$-phase and $\beta$-phase particles form intrinsically when colloids of $\alpha$-phase particles are heated for a sufficiently long time, because seeds of the $\beta$-phase nucleate in the solution after some time at high temperatures. Since the number of seeds determines the final size of the $\beta$-phase product, control of the nucleation is crucial for controlling the final particle size. We show that the number of $\beta$-phase seeds strongly depends on the composition of the $\alpha$-phase known to form solid solutions $\mathrm{Na}_{1-x} \mathrm{YF}_{4-x}$ in the range from $x=0$ to $x=4 / 9$. Sodium-deficient $\alpha$-phase particles form a negligible number of $\beta$-phase seeds whereas $\alpha$-phase particles with high sodium content yield a very large number of seeds. By taking advantage of this dependence and modifying the synthesis of the $\alpha$-phase particles accordingly, small phase-pure $\beta-\mathrm{NaYF}_{4}: \mathrm{Yb}, \mathrm{Er}$ particles with a size smaller than $6 \mathrm{~nm}$ can be prepared in oleic acid/octadecene just as well as much larger particles.
\end{abstract}

\section{Introduction}

Upconversion nanocrystals have applications in biological labelling/sensing and imaging, ${ }^{1-10}$ photodynamic therapy, ${ }^{1,11}$ spectral conversion in solar cells, ${ }^{12,13} 3 \mathrm{D}$ optical displays, ${ }^{14-17}$ optical storage, ${ }^{18}$ lasing $^{19,20}$ and security printing. ${ }^{21-24} \mathrm{~A}$ variety of synthetic methods have been described for producing solvent-dispersible sodium rare-earth tetrafluoride nanocrystals with controlled size, shape and capping ligands. Most of these procedures are based on thermal decomposition, ${ }^{25-31}$ co-precipitation, ${ }^{32-41}$ or solvothermal methods. ${ }^{4,16,42-49}$ In many of these methods oleic acid containing solvent mixtures have been used yielding nanocrystals of high quality and with very narrow particle size distributions. ${ }^{4,6,10,17,21-27,29,32-34,37,41,44}$ The formation of upconversion particles of the hexagonal $\beta$-phase has already been proved to be affected by several synthesis parameters, such as the concentration of sodium salts, ${ }^{25,27,31,32,41,44,46,48,49}$ the concentration of metal oleates, ${ }^{31}$ the choice of the fluoride source, ${ }^{43}$ the doping level ${ }^{17,48}$ and the choice of the solvent mixture..$^{27,28,32,34,44}$ Most synthesis procedures for the $\beta$-phase $\mathrm{NaYF}_{4}, \mathrm{NaYbF}_{4}, \mathrm{NaErF}_{4}$, and $\mathrm{NaYF}_{4}: \mathrm{Yb}, \mathrm{Er}$ yield monodisperse particles with a mean size

Institute of Chemistry of New Materials, Osnabrück University, Barbarastraße 7, 49076 Osnabrück, Germany. E-mail: markus.haase@uni-osnabrueck.de;

Fax: (+49) 541 969-3233

$\dagger$ Electronic supplementary information (ESI) available. See DOI: 10.1039/c4nr03833a between 15 and $100 \mathrm{~nm} .^{6,17,27}$ This is in contrast to other $\mathrm{NaREF}_{4}$ (RE $=$ rare-earth) nanomaterials ${ }^{50,51}$ such as, for example, $\mathrm{NaGdF}_{4}, \mathrm{NaEuF}_{4}, \mathrm{NaTbF}_{4}, \mathrm{NaSmF}_{4}$ (group II materials, according to $\mathrm{Mai}^{27}$ ), where similar reaction conditions yield particles in the sub-10 nm size regime. In fact, sub-10 nm particles $\mathrm{NaYF}_{4}: \mathrm{Yb}$,Er were successfully prepared by replacing part of the yttrium by gadolinium ${ }^{17,52,53}$ and by doping $\mathrm{NaYF}_{4}$ with Eu and $\mathrm{Tb}^{54}$ To the best of our knowledge, however, only two papers report on the successful synthesis of nearly monodisperse $\beta-\mathrm{NaYF}_{4}: \mathrm{Yb}, \mathrm{Er}$ particles with sizes below $10 \mathrm{~nm}$ without additional doping. ${ }^{32,55}$ The smallest $\mathrm{NaYF}_{4}$ : Yb,Er particles with diameters down to $4.5 \mathrm{~nm}$ were described by Ostrowski et al. ${ }^{32}$

Studies that unravel the parameters which determine the final size of $\beta-\mathrm{NaREF}_{4}$ nanocrystals are complicated by the fact that not only particles of the hexagonal $\beta$-phase but also those of the cubic $\alpha$-phase can nucleate and grow during the decomposition of the molecular precursors. For several synthesis procedures it has been shown that $\alpha$-phase particles are formed as the first product which subsequently convert to pure $\beta$-phase particles as the final material. ${ }^{25,44,56,57}$ The question then is by what mechanism nearly monodisperse $\beta$-phase particles can grow from small $\alpha$-phase particles as the Ostwaldripening of small particles in the absence of additional monomer precursors should yield broad size distributions. ${ }^{58-60}$ Narrow particle size distributions usually result from focussing of the size distribution which requires that a high supersatura- 
tion of monomers is maintained during particle growth. ${ }^{58,61}$ The latter requires a high production rate of monomers in solution, which is achieved in thermal decomposition methods for $\mathrm{NaREF}_{4}$ nanocrystals by employing metal trifluoroacetates as thermally labile precursors for the fluoride and metal ions. ${ }^{25-31}$ Mai et al. thoroughly investigated the reaction mechanisms for this method and showed that also in this case $\alpha$-phase $\mathrm{NaYF}_{4}$ nanocrystals are formed as the first product. $^{25,27}$ Zhang et al. introduced a variation of the co-precipitation method using lanthanide chlorides, $\mathrm{NaOH}$, and $\mathrm{NH}_{4} \mathrm{~F}$ as precursors. ${ }^{33,34}$ In their method the precursors are not injected into a hot solution but the reactants are combined at room temperature and subsequently heated. Nevertheless, $\beta-\mathrm{NaYF}_{4}$ nanocrystals of uniform size distribution are obtained. Similar to Mai et al. they showed that the synthesis of $\beta-\mathrm{NaYF}_{4}$ nanocrystals occurs via an intermediate formation of small $\alpha$-phase particles which are subsequently converted to larger $\beta$-phase nanocrystals. ${ }^{25,34}$ Furthermore, Mai et al. have shown that $\beta$-phase particles with narrow particle size distributions are even obtained if the $\alpha$-phase particles are isolated, purified and heated in oleic acid/octadecene in the absence of any additional precursor. ${ }^{25,62}$ The latter result raises the question whether the $\beta$-phase particles grow at all by Ostwald-ripening, that is, by the exchange of monomers. To investigate this issue we have recently studied the growth behaviour of particle mixtures containing small $\alpha-\mathrm{NaYF}_{4}$ and small $\alpha-\mathrm{NaGdF}_{4}$ particles as well as mixtures containing small $\alpha-\mathrm{NaEuF}_{4}$ and small $\alpha-\mathrm{NaGdF}_{4}$ particles. $^{51,56}$ Luminescence spectroscopy and EPR measurements show that the resulting monodisperse particles of $\beta-\mathrm{NaGdF}_{4}: \mathrm{Eu}$ and $\beta-\mathrm{NaYF}_{4}: \mathrm{Gd}$ mainly contain isolated dopant ions. The small $\mathrm{NaEuF}_{4}$ and $\mathrm{NaGdF}_{4}$ particles used as a source for the dopant ions were therefore not incorporated into the final $\beta$-phase particles as whole building blocks. Instead, the separation of the dopant ions in the crystal lattice of the product indicates that the small particles dissolve during particle growth, in accord with the mechanism of Ostwald-ripening. $\mathrm{NaGdF}_{4}$ and $\mathrm{NaEuF}_{4}$ have the additional advantage that not only small particles of the $\alpha$-phase but also small particles of the $\beta$-phase can be prepared, allowing study of the growth behaviour of small particles of both phases separately. The results show that the small $\beta$-phase particles behave as expected for Ostwald-ripening, that is, they show broadening of the size distribution. Closer inspection of the particle growth by TEM reveals a weak broadening of the size distribution in the case of the $\alpha$-phase particles, too, but only at early growth stages, before seeds of the $\beta$-phase nucleate in solution. $^{25,51}$ After nucleation of these $\beta$-phase seeds, the $\alpha$-phase particles rapidly dissolve whereas the $\beta$-phase seeds rapidly increase in size. ${ }^{51,63}$ This observation can be explained by a higher solubility of the thermodynamically less stable $\alpha$-phase. The release of monomers during the fast dissolution of the $\alpha$-phase results in a monomer concentration so high that particles of the less soluble $\beta$-phase grow under the conditions of monomer supersaturation. Numerical simulations based on the LSW-theory of Ostwald-ripening confirm this model and show that the Ostwald-ripening of such binary par- ticle mixtures in fact results in fast dissolution of the $\alpha$-phase and focussing of the particle size distribution of the $\beta$-phase. ${ }^{63}$

In the present paper we have extended our study on the growth behaviour of small $\mathrm{NaREF}_{4}$ nanocrystals to the upconversion material $\mathrm{NaYF}_{4}: \mathrm{Yb}, \mathrm{Er}$ and its undoped host lattice $\mathrm{NaYF}_{4}$. Again, purified $\alpha$-phase particles were used as singlesource precursors to prepare the final particles. The latter are thereby formed in the absence of any molecular precursors apart from the monomers exchanged among the particles during Ostwald-ripening. This "clean" reaction solely based on Ostwald-ripening makes it easier to identify the conditions required to obtain $\beta$-phase particles of small size. In contrast to our earlier work on $\mathrm{NaSmF}_{4}, \mathrm{NaEuF}_{4}, \mathrm{NaGdF}_{4}$, and $\mathrm{NaTbF}_{4}$, this paper is focussed on the different compositions of the cubic $\alpha$-phase known to form solid solutions $\mathrm{Na}_{1-x} \mathrm{YF}_{4-x}$ in the range from $x=0$ to $x=4 / 9$. We have therefore varied the ratio of sodium and rare-earth metal in the synthesis of the $\alpha$-phase particles accordingly and have used the resulting particles, after washing and redispersing them in pure oleic acid/octadecene solvent, to prepare $\beta$-phase particles. We show that the ratio of sodium and rare-earth metal is one of the key parameters determining the final size of the $\beta$-phase particles, since the ratio affects the number of $\beta$-phase seeds that nucleate during Ostwald-ripening of the $\alpha$-phase particles. By adjusting this parameter we are able to reproducibly prepare $\beta-\mathrm{NaYF}_{4}: \mathrm{Yb}, \mathrm{Er}$ particles in oleic acid/octadecene with a size smaller than $6 \mathrm{~nm}$.

\section{Experimental}

\section{Materials}

Sodium oleate (82\%, Sigma-Aldrich), ammonium fluoride (98\%, Sigma-Aldrich), oleic acid (90\%, Alfa Aesar), octadecene (90\%, Alfa Aesar) and hydrated rare-earth chlorides of $\mathrm{YCl}_{3}$, $\mathrm{YbCl}_{3}$ and $\mathrm{ErCl}_{3}$ (99.9\%, Treibacher Industrie AG) were used as received.

\section{Rare-earth oleates}

The rare-earth oleates were prepared by reacting solutions of the corresponding rare-earth chlorides with sodium oleate, as given in the literature. ${ }^{64}$ In a typical preparation of yttrium oleate, $60 \mathrm{mmol}$ yttrium chloride $(18.2 \mathrm{~g})$ and $180 \mathrm{mmol}$ sodium oleate $(54.8 \mathrm{~g}$ ) were dissolved in $120 \mathrm{ml}$ ethanol, $80 \mathrm{ml}$ water and $210 \mathrm{ml}$ hexane. The resulting turbid solution was heated for $14 \mathrm{~h}$ under reflux (at about $60^{\circ} \mathrm{C}$ ). The transparent organic phase containing the yttrium oleate was separated at room temperature and the hexane was removed with a rotavap, yielding yttrium oleate. Ytterbium oleate and erbium oleate were prepared analogously.

\section{Synthesis of small cubic phase nanocrystals}

The synthesis of the sub-10 nm particles of $\alpha-\mathrm{NaREF}_{4}(\mathrm{RE}=\mathrm{Y}$, $\mathrm{Yb}$ and $\mathrm{Er}$ ) is based on the reaction of rare-earth oleates, sodium oleate and $\mathrm{NH}_{4} \mathrm{~F}$ in a mixture of oleic acid and octadecene, similar to the procedure given by Liu et $a{ }^{41}$ In our case, 
the rare-earth oleate and the sodium oleate were combined in different molar ratios of 1 to $x, x$ ranging from $5 / 9$ to 8 . Oleic acid and octadecene were added to the metal oleates $(10 \mathrm{ml}$ of each solvent per $1 \mathrm{mmol}$ of rare-earth oleates) and the mixture was degassed on a vacuum Schlenk-line $(1 \mathrm{mbar})$ for $1 \mathrm{~h}$ at $100{ }^{\circ} \mathrm{C}$ under vigorous stirring. Then $3+x \mathrm{mmol}$ of $\mathrm{NH}_{4} \mathrm{~F}$ was added and the apparatus was cycled three times between nitrogen and vacuum. The reaction mixture was heated at $200{ }^{\circ} \mathrm{C}$ for $60 \mathrm{~min}$ under nitrogen flow and vigorous stirring and thereafter allowed to cool at room temperature. When an excess of sodium oleate and ammonium fluoride was used, sodium fluoride was formed as a by-product which was removed by centrifugation. The clear supernatant was merged with an equal volume of ethanol leading to precipitation of the nanoparticles, which were separated by centrifugation. The particles were purified by redispersing the precipitate in hexane ( $3 \mathrm{ml}$ hexane per $1 \mathrm{mmol}$ rare-earth oleates), followed by precipitation with ethanol and separation by centrifugation.

Yb,Er-doped up-conversion nanoparticles were prepared similarly by using yttrium, ytterbium and erbium in the usual molar ratio of $0.78: 0.20: 0.02$.

\section{Synthesis of hexagonal phase nanocrystals}

For the preparation of $\beta-\mathrm{NaREF}_{4}$ particles $(\mathrm{RE}=\mathrm{Y}, \mathrm{Yb}, \mathrm{Er})$, small $\alpha$-phase particles were dissolved in a mixture of oleic acid and octadecene $(2 \mathrm{ml}$ of oleic acid and $2 \mathrm{ml}$ of octadecene per $1 \mathrm{mmol}$ of cubic phase particles). The mixture was degassed on a vacuum Schlenk-line $(1 \mathrm{mbar})$ for $1 \mathrm{~h}$ at $100{ }^{\circ} \mathrm{C}$ under vigorous stirring. The resulting clear colloidal solution was heated at $300{ }^{\circ} \mathrm{C}$ for different periods of time under nitrogen flow and vigorous stirring. During the growth reaction several samples (each $5 \mathrm{ml}$ ) were taken with a glass syringe and injected into a cooled mixture of hexane and ethanol ( $2 \mathrm{ml}$ of hexane and $4 \mathrm{ml}$ of ethanol per $\mathrm{ml}$ sample). The precipitated nanocrystals were separated by centrifugation and purified as given above for particles of the cubic phase.

\section{Instrumentation}

TEM-images were recorded with a JEM 2100 electron microscope (JEOL) operated at $200 \mathrm{kV}$. Size histograms of the samples were derived from TEM images using the software Image-J. ${ }^{65}$ In the case of rod-shaped particles, the length and the width of each particle were determined from the TEM images and used to calculate the volume of each particle assumed to have cylindrical shape. The diameter of a sphere with identical volume was then calculated from this value and used for the histogram. X-ray powder diffraction measurements were performed on an X'Pert Pro Diffractometer (Panalytical) with Bragg-Brentano geometry using $\mathrm{Cu} \mathrm{K}_{\alpha}(\lambda=$ $1.5406 \AA)$ radiation $(40 \mathrm{kV}, 40 \mathrm{~mA})$ and a $2 \theta$ step size of $0.0334^{\circ}$. The XRD data of all samples were analyzed by the Rietveld method using the software FullProf (version July 2011. LLB, Juan Rodriguez Carvajal, Saclay France). ${ }^{66}$ The elemental composition of samples was determined by X-ray fluorescence analysis (XFA) using a $1 \mathrm{~kW}$ AXIOS $^{\mathrm{mAX}}$ X-ray fluorescence spectrometer (Panalytical).

\section{Results and discussion}

The growth behaviour of sub-10 nm $\mathrm{NaYF}_{4} ; \mathrm{Yb}, \mathrm{Er}$ upconversion nanocrystals was studied by redispersing small particles of the cubic $\alpha$-phase and the hexagonal $\beta$-phase in oleic acid/octadecene solvent and heating the resulting colloids at $300^{\circ} \mathrm{C}$. The $\alpha$-phase nanocrystals were purified before further use by re-dispersing the particles in hexane and precipitating the nanocrystals again with ethanol. Different molar ratios of sodium oleate, rare-earth oleate and ammonium fluoride were used in the synthesis of the $\alpha$-phase starting material, as it is well known that the cubic phase of bulk $\mathrm{NaYF}_{4}, \mathrm{NaYbF}_{4}$ and $\mathrm{NaErF}_{4}$ forms solid solutions allowing to adjust the composition $\mathrm{Na}_{1-x} \mathrm{REF}_{4-x}$ between $x=0$ and $x=4 / 9 .{ }^{67,68}$

To obtain sub-10 nm $\mathrm{NaYF}_{4}: \mathrm{Yb}, \mathrm{Er}$ particles of the hexagonal $\beta$-phase as starting material we tested a procedure analogous to the one used in our previous paper for the synthesis of small particles of $\beta-\mathrm{NaSmF}_{4}, \beta-\mathrm{NaEuF}_{4}, \beta-\mathrm{NaGdF}_{4}$ and $\beta-\mathrm{NaTbF}_{4}$. In the case of $\mathrm{NaYF}_{4}, \mathrm{NaYbF}_{4}$ and $\mathrm{NaErF}_{4}$, however, this method yields nanocrystals of the $\alpha$-phase. Therefore, we used a new synthesis procedure based on $\alpha$-phase particles with high sodium content as the precursor material. The details of this synthesis method will be given below.

TEM images of three different starting materials are displayed in the upper part of Fig. 1. The figure shows small $\alpha-\mathrm{NaYF}_{4}: \mathrm{Yb}, \mathrm{Er}$ particles prepared with an excess of sodium and fluoride (molar ratio of $2.5: 1: 4$ with respect to sodium oleate, rare-earth oleate and ammonium fluoride), small sodium-deficient particles of the cubic $\alpha$-phase with nominal composition $\mathrm{Na}_{5} \mathrm{Y}_{9} \mathrm{~F}_{32}: \mathrm{Yb}$,Er, as well as small $\mathrm{NaYF}_{4}: \mathrm{Yb}$,Er particles of the hexagonal $\beta$-phase. Before heating to $300{ }^{\circ} \mathrm{C}$ all materials show mean particles sizes well below $10 \mathrm{~nm}$ and narrow size distributions (Fig. 1, upper part). After heating (lower part), broad size distributions are observed for $\alpha-\mathrm{Na}_{5} \mathrm{Y}_{9} \mathrm{~F}_{32}: \mathrm{Yb}$,Er and $\beta-\mathrm{NaYF}_{4}: \mathrm{Yb}$,Er particles but a narrow size distribution is observed in the case of $\alpha-\mathrm{NaYF}_{4}: \mathrm{Yb}, \mathrm{Er}$ as the starting material. The corresponding XRD data and size histograms are displayed in Fig. S1 and S2, respectively (ESI $\dagger$ ). The $\mathrm{XRD}$ data show that the crystal structure of the $\alpha-\mathrm{NaYF}_{4}: \mathrm{Yb}, \mathrm{Er}$ particles changes from cubic ( $\alpha$-phase) to hexagonal ( $\beta$-phase) during heating whereas no phase transition is observed for the $\beta$-phase and the sodium-deficient $\alpha$-phase material. The histograms show that nearly monodisperse particles are only obtained when the phase transition is also observed. The growth of the other two materials yields the broad size distributions expected for Ostwald-ripening in the absence of additional monomers. These observations are in accord with our earlier results for $\mathrm{NaSmF}_{4}, \mathrm{NaEuF}_{4}, \mathrm{NaGdF}_{4}$ and $\mathrm{NaTbF}_{4}$ nanocrystals where narrow size distributions also require that a transition from the $\alpha$-phase to the $\beta$-phase takes place. ${ }^{51}$

The conversion of small $\alpha-\mathrm{NaYF}_{4}: \mathrm{Yb}, \mathrm{Er}$ particles to larger particles of the $\beta$-phase is shown in more detail in Fig. 2 where the mean sizes of the $\alpha$-phase and the $\beta$-phase particles as well as the molar fractions of the two phases are plotted versus reaction time. The figure is based on Rietveld fits of the XRD data of $\mathrm{NaYF}_{4}: \mathrm{Yb}$,Er samples taken after different times of growth 


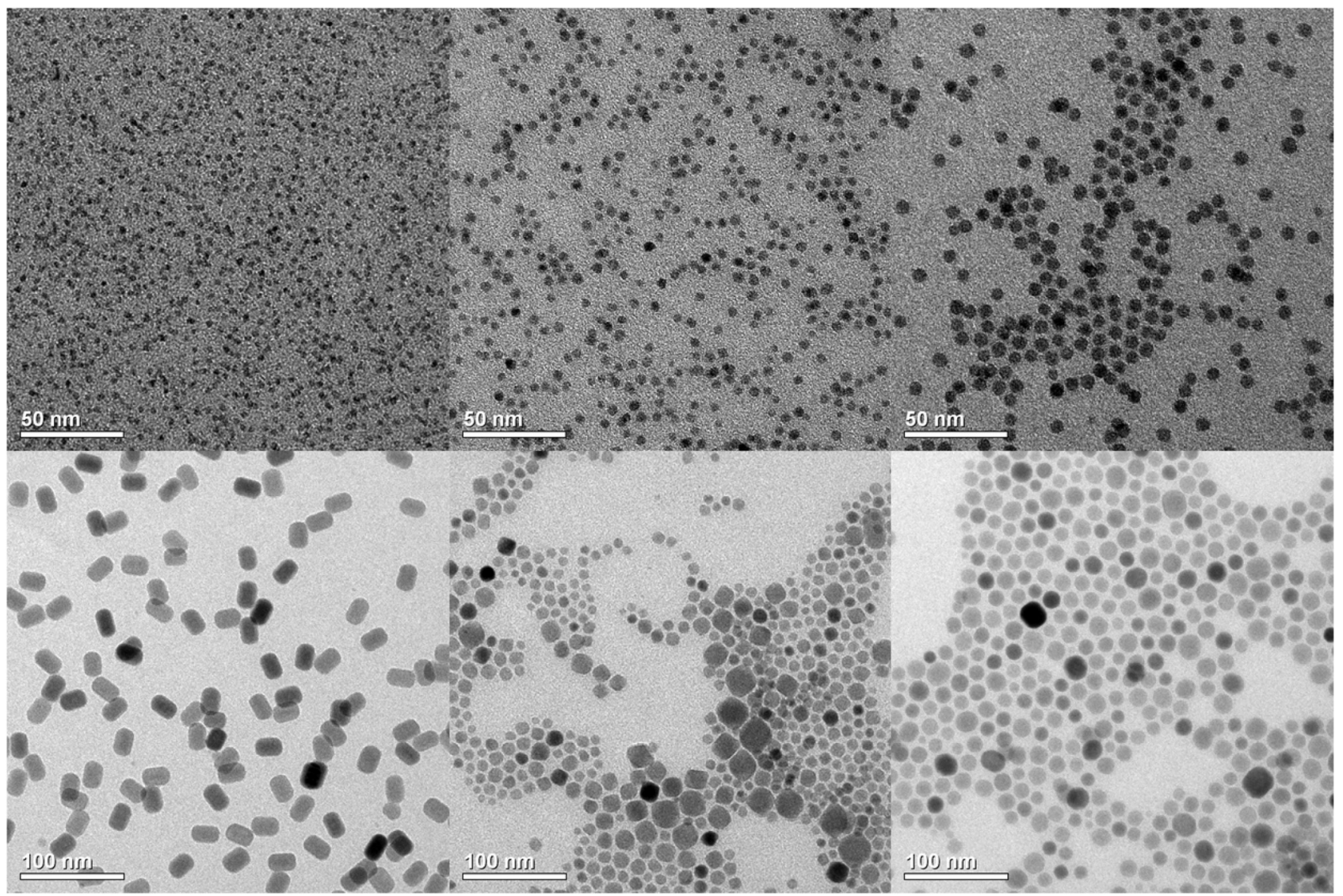

Fig. 1 Upper row: TEM-images of sub-10 nm nanocrystals of $\alpha-\mathrm{NaYF}_{4}: \mathrm{Yb}, \mathrm{Er}, \alpha-\mathrm{Na}_{5} \mathrm{Y}_{9} \mathrm{~F}_{32}: \mathrm{Yb}, \mathrm{Er}$, and $\beta-\mathrm{NaYF}$ : $\mathrm{Yb}$, Er (from left to right). Lower row: particles of the upper row after Ostwald-ripening in oleic acid/octadecene for $80 \mathrm{~min}$ at $300{ }^{\circ} \mathrm{C}$. Particles with narrow size distribution are only obtained by heating $\alpha-\mathrm{NaYF}_{4}: \mathrm{Yb}$,Er particles, the only case where Ostwald-ripening is accompanied by a transition from the $\alpha$-phase to the $\beta$-phase.

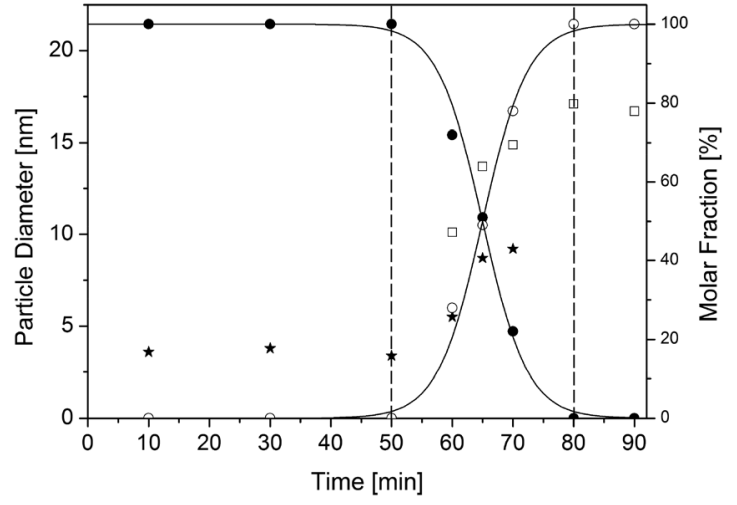

Fig. 2 Ostwald-ripening of sub-10 nm nanocrystals of $\alpha-\mathrm{NaYF}_{4}: \mathrm{Yb}, \mathrm{Er}$ in oleic acid/octadecene at $300{ }^{\circ} \mathrm{C}$. The figure displays the temporal evolution of the mean size of the $\alpha$-phase particles (filled stars), the mean size of $\beta$-phase particles nucleating in the solution (open cubes) and the molar fractions of the two phases (circles and lines). A molar ratio of $2.5: 1: 4$ (Na-oleate : $\mathrm{RE}$-Oleate : $\mathrm{NH}_{4} \mathrm{~F}$ ) was used in the synthesis of the $\alpha$-phase particles.

at $300{ }^{\circ} \mathrm{C}$ (see Fig. $\mathrm{S} 3$ and $\mathrm{S} 4 \uparrow$ for the XRD data and a selection of Rietveld fits). First weak reflexes of the hexagonal $\beta$-phase can be identified in the XRD data after 60 min of heating at $300^{\circ} \mathrm{C}$. This delayed nucleation of the $\beta$-phase was investigated by Mai et al. and was attributed to a high activation energy barrier for the transition of the $\alpha$-phase to the $\beta$-phase. ${ }^{25,27}$ The figure shows that the particles of the $\alpha$-phase rapidly dissolve as soon as a sufficient number of $\beta$-phase particles nucleated in solution. The rapid dissolution is accompanied by a fast increase of the molar fraction of the $\beta$-phase as also observed by other groups. ${ }^{25,27,31,42,63}$ Obviously, the monomers released by the rapidly dissolving $\alpha$-phase particles are consumed by the $\beta$-phase seeds. In fact, the numerical simulations mentioned above show that the thermodynamically less stable $\alpha$-phase itself act as monomer reservoir which releases monomers at a high rate. ${ }^{58,61}$ The simulations not only reproduce the strong focussing of the size distribution observed for all these systems but also the temporal evolution of the mean particle sizes and molar fractions. ${ }^{63}$

Small particles of undoped $\mathrm{NaYF}_{4}$ show a growth behaviour analogous to that given in Fig. 2 for the doped material confirming that doping is not a key factor for obtaining narrow size distributions (Fig. S5 $†$ ). Fig. 3 shows that heating of undoped particles of $\alpha-\mathrm{NaErF}_{4}, \alpha-\mathrm{NaYbF}_{4}$ or $\alpha-\mathrm{NaYF}_{4}$ particles in oleic acid/octadecene yields in fact narrow size distributions in all cases whereas broad size distributions are observed for the corresponding sodium-deficient systems $\alpha-\mathrm{Na}_{5} \mathrm{Er}_{9} \mathrm{~F}_{32}$, $\alpha-\mathrm{Na}_{5} \mathrm{Yb}_{9} \mathrm{~F}_{32}$, and $\alpha-\mathrm{Na}_{5} \mathrm{Y}_{9} \mathrm{~F}_{32}$ where no phase transition occurs 


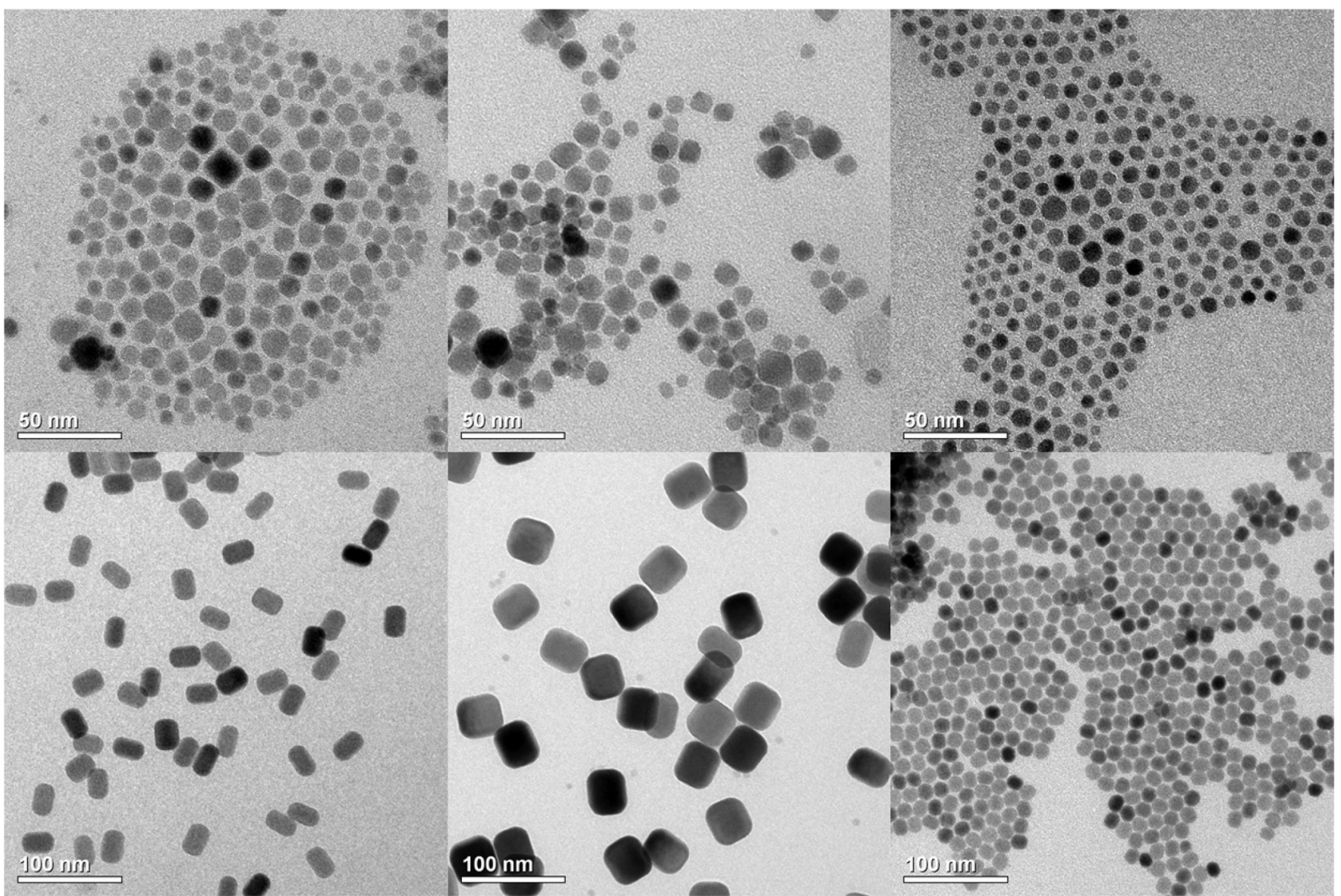

Fig. 3 Upper row: TEM-images of the polydisperse $\alpha$-phase products obtained by heating sub-10 nm particles of $\alpha-\mathrm{Na}_{5} \mathrm{Y}_{9} \mathrm{~F}_{32}, \alpha-\mathrm{Na}_{5} \mathrm{Yb}_{9} \mathrm{~F}_{32},{ }$ and $\alpha-\mathrm{Na}_{5} \mathrm{Er}_{9} \mathrm{~F}_{32}$ at $300{ }^{\circ} \mathrm{C}$ in oleic acid/octadecene (from left to right). Lower row: nearly monodisperse $\beta$-phase products obtained by heating sub-10 nm particles of $\alpha-\mathrm{NaYF}_{4}, \alpha-\mathrm{NaYbF}_{4}$, and $\alpha-\mathrm{NaErF}_{4}$ at $300{ }^{\circ} \mathrm{C}$ in oleic acid/octadecene (from left to right). The sub-10 nm particles of $\alpha-\mathrm{Na}_{5} \mathrm{RE}_{9} \mathrm{~F}_{32}$ and $\alpha-\mathrm{NaREF}_{4}$ were prepared by employing Na-OA, RE-OA and $\mathrm{NH}_{4} \mathrm{~F}$ in a molar ratio of $5: 9: 32$ and $2.5: 1: 4$, respectively.

(see also Fig. S6 and S7†). Obviously, the sodium content of the cubic material affects the nucleation of $\beta$-phase seeds in all cases. We therefore studied the influence of the molar ratio of $\mathrm{NaF}$ and rare-earth trifluoride by varying the molar ratios of sodium oleate, rare-earth oleate and ammonium fluoride from $8: 1: 11$ to $0.56: 1: 3.56$ in the synthesis of the $\alpha$-phase starting material. After heating the three components in oleic acid/ octadecene at $200{ }^{\circ} \mathrm{C}$ the resulting $\alpha$-phase upconversion particles were precipitated with ethanol and thereafter washed by re-dispersing the precipitate in hexane, adding again ethanol and isolating the particles by centrifugation. The XRD data of the resulting powders show the expected signals of the $\alpha$-phase in all cases but no signals of NaF indicating that the latter is mostly removed by the washing sequence (Fig. S $8 \dagger$ ). The composition of the different $\alpha$-phase particles was determined by X-ray fluorescence analysis (XRF). In Fig. 4 the ratio of sodium to rare-earth metal in the purified particles, as determined by $\mathrm{XRF}$, is plotted versus the ratio of sodium to rare-earth oleate employed in the synthesis of the particles. The figure shows that $\alpha$-phase particles prepared with a low ratio of sodium to rare-earth oleate display the expected sodium-deficient composition. If the particles are prepared with a high ratio of sodium to rare-earth oleate, the $\alpha$-phase particles contain up to $20 \%$

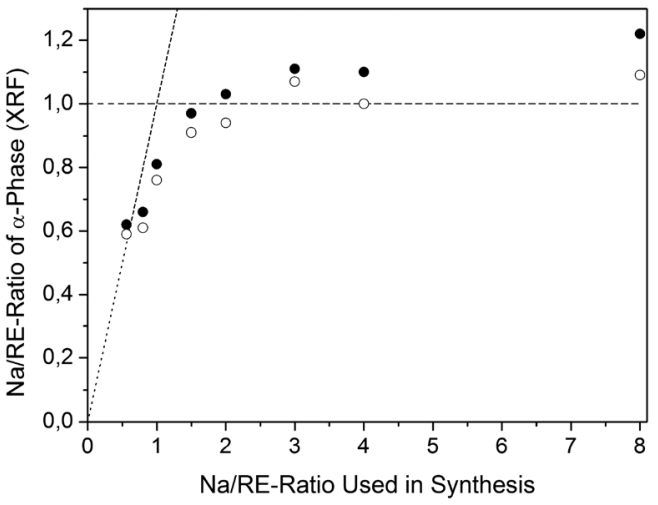

Fig. 4 X-ray fluorescence (XRF) analysis data of $\alpha-\mathrm{NaYF}_{4}: \mathrm{Yb}, \mathrm{Er}$ particles prepared with different amounts of sodium oleate ( $\mathrm{Na}-\mathrm{OA})$, rare-earth oleate $(\mathrm{RE}-\mathrm{OA})$ and ammonium fluoride $\left(\mathrm{NH}_{4} \mathrm{~F}\right)$ before (filled circles) and after (open circles) washing. The ratio of sodium to rare-earth metal in the particles, as determined by $\mathrm{XRF}$, is plotted versus the ratio of sodium to rare-earth oleate employed in the synthesis of the particles.

excess sodium with respect to the expected $1: 1$ ratio of sodium to rare-earth metal. This excess of sodium, however, can be reduced by extensive washing of the particles (Fig. 4). Since extensive washing also negatively affects the dispersibility 
of the particles we assign this additional sodium to the presence of sodium oleate in the ligand shell of the particle surface or as ad-atoms on the particle surface. ${ }^{69}$

The purified $\alpha$-phase particles were then re-dispersed in oleic acid/octadecene solvent and heated to $300{ }^{\circ} \mathrm{C}$. The conversion of the $\alpha$-phase to the $\beta$-phase with time is shown in Fig. 5 for the different $\alpha$-phase materials. The figure shows that with increasing sodium-deficiency of the $\alpha$-phase the delay of the conversion to the $\beta$-phase also increases. Highly sodium-deficient $\alpha$-phase particles (5:9:32 molar ratio of $\mathrm{Na}-\mathrm{OA}, \mathrm{RE}-\mathrm{OA}$ and $\mathrm{NH}_{4} \mathrm{~F}$ ) do not convert to the $\beta$-phase even after 4 hours of heating, as already shown in Fig. 3. This increasing delay shows that the sodium content of the $\alpha$-phase particles affects the nucleation of the $\beta$-phase seeds, because the dissolution of the $\alpha$-phase does not start before the first $\beta$-phase seeds have formed (see also Fig. 2 and S5†). This is even more evident when the sizes of the resulting $\beta$-phase particles are compared, because the size of the particles is inversely proportional to the number of seeds formed. Fig. 6 and 7

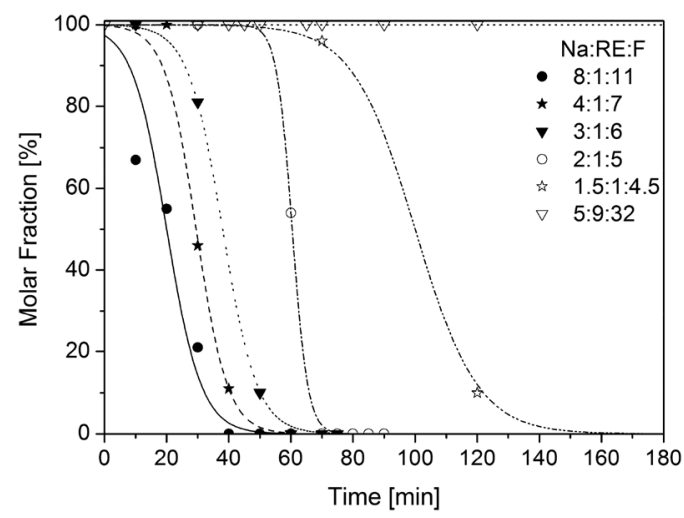

Fig. 5 Conversion of different $\alpha-\mathrm{NaYF}_{4}: \mathrm{Yb}, \mathrm{Er}$ particles to $\beta-\mathrm{NaYF}_{4}: \mathrm{Yb}, \mathrm{Er}$ particles with time. With increasing sodium deficiency of the $\alpha$-phase particles the conversion to the $\beta$-phase is increasingly delayed. Highly sodium-deficient $\alpha$-phase particles do not convert to the $\beta$-phase within 4 hours of heating. The molar ratios ( $\mathrm{Na}-\mathrm{OA}$ : $\mathrm{RE}-\mathrm{OA}: \mathrm{NH}_{4} \mathrm{~F}$ ) given in the diagram refer to the ratios used in the synthesis of the $\alpha$-phase particles.

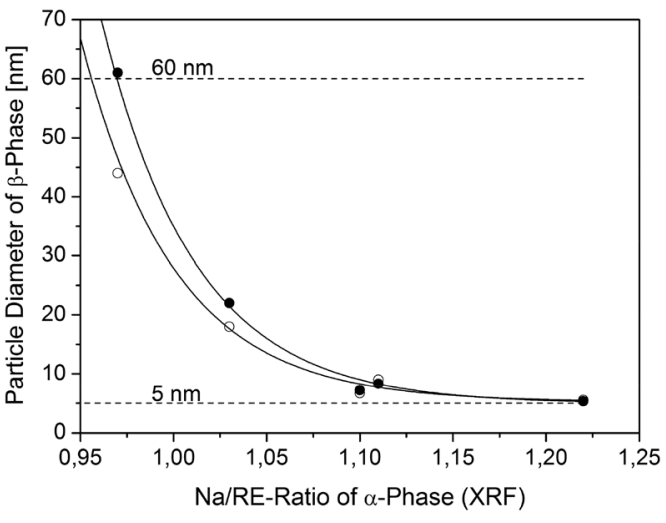

Fig. 7 Mean size of nearly monodisperse $\beta-\mathrm{NaYF}_{4}: \mathrm{Yb}, \mathrm{Er}$ particles prepared by heating $\alpha$-phase particles with different compositions in oleic acid/octadecene at $300{ }^{\circ} \mathrm{C}$. The Na:RE-ratio of the $\alpha$-phase particles was determined by $\mathrm{X}$-ray fluorescence spectroscopy (XRF). The $\alpha$-phase particles were heated either in the presence (open circles) or in the absence (filled circles) of additional sodium oleate and $\mathrm{NH}_{4} \mathrm{~F}$ in the solvent.

show that the particle size of the nearly monodisperse $\beta$-phase systematically decreases with increasing sodium content of the $\alpha$-phase particles (see also Fig. S9-S11†). In fact, $\beta$-phase $\mathrm{NaYF}_{4}: \mathrm{Yb}, \mathrm{Er}$ particles with a mean size as small as $5.3 \mathrm{~nm}$ can be reproducibly prepared in gram amounts by heating $\alpha$-phase particles prepared with a high molar ratio of $8: 1: 11$ (Na-OA: RE-OA: $\mathrm{NH}_{4} \mathrm{~F}$ ). Similar results were obtained for undoped $\mathrm{NaYF}_{4}$ (see Fig. S14-S17†). Note that in our case the particles are synthesized in the absence of oleylamine which is often used to favour the formation of the cubic $\alpha$-phase. The small $\beta$-phase upconversion particles prepared in this way were used for the Ostwald-ripening experiments shown in Fig. 1. When the ratio of the three components is reduced to $2: 1: 5$, the resulting $\alpha$-phase particles yield $\beta$-phase particles with a mean size of $22 \mathrm{~nm}$. If the ratio is further reduced, also the particle size of the $\beta$-phase increases further, but at very low sodium contents the conversion to the $\beta$-phase becomes incomplete and finally does not take place anymore. Note that according

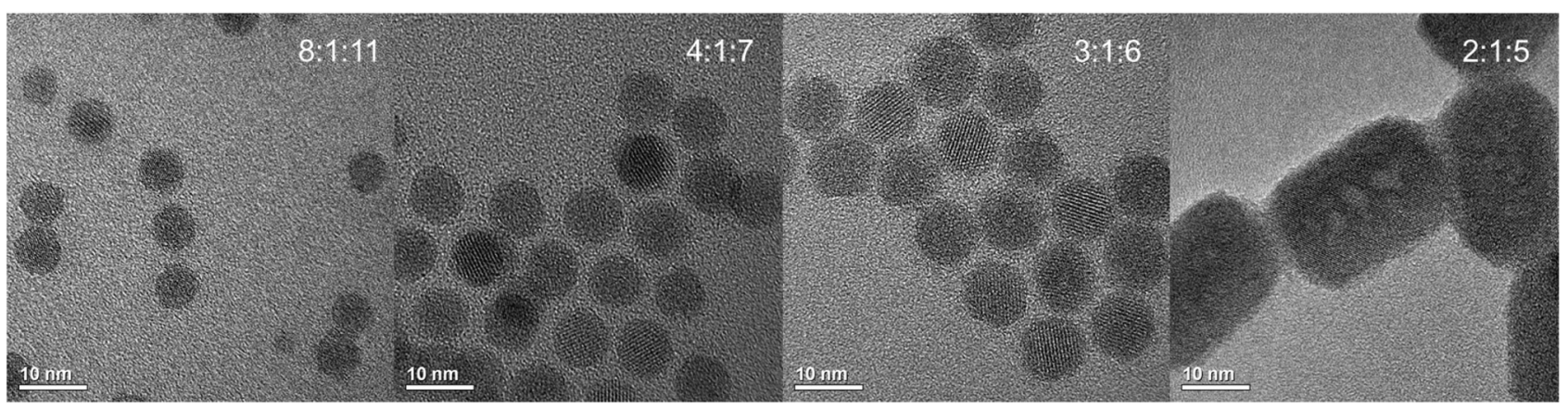

Fig. $6 \beta-\mathrm{NaYF}_{4}: \mathrm{Yb}$,Er particles with different mean size prepared by Ostwald-ripening of purified $\alpha-\mathrm{NaYF}$ :Yb,Er particles in oleic acid/octadecene at $300{ }^{\circ} \mathrm{C}$. The number of $\beta$-phase seeds nucleating during Ostwald-ripening of the $\alpha$-phase particles depends on the molar ratio of sodium oleate $(\mathrm{Na}-\mathrm{OA})$, rare-earth oleate (RE-OA) and ammonium fluoride $\left(\mathrm{NH}_{4} \mathrm{~F}\right)$ employed in the synthesis of the $\alpha$-phase particles. The molar ratio $(\mathrm{Na}-\mathrm{OA}$ : $\mathrm{RE}-\mathrm{OA}: \mathrm{NH}_{4} \mathrm{~F}$ ) is given in each TEM-image. The different mean sizes of the $\beta$ - $\mathrm{NaYF}_{4}: \mathrm{Yb}, \mathrm{Er}$ particles are also given in Fig. 7, overview TEM-images and size histograms are shown in the ESI. $\dagger$ 
to the phase diagrams of bulk $\mathrm{NaYF}_{4}, \mathrm{NaYbF}_{4}$ and $\mathrm{NaErF}_{4}$, the cubic $\alpha$-phase is stable for compositions ranging from $\mathrm{NaYF}_{4}$ to $\mathrm{Na}_{5 / 9} \mathrm{YF}_{32 / 9}$ whereas the $\beta$-phase is only stable in a narrow range close to the $1: 1$ composition given by the formula $\mathrm{NaYF}_{4} \cdot{ }^{67,70}$ Strongly sodium-deficient $\alpha$-phase particles should therefore not contain enough sodium fluoride to completely convert to the $\beta$-phase. For this reason we have also studied the conversion of the $\alpha$-phase particles in the presence of additional sodium oleate and $\mathrm{NH}_{4} \mathrm{~F}$ in solution. A rather large amount of $0.6 \mathrm{mmol}$ of $\mathrm{Na}-\mathrm{OA}$ and $0.6 \mathrm{mmol}$ of $\mathrm{NH}_{4} \mathrm{~F}$ per $1 \mathrm{mmol}$ of nanoparticles in oleic acid/octadecene was used in these experiments which exceeded the amount of Na-OA contained in the ligand shell in all cases (Fig. 4). The result is that in the presence of additional sodium oleate and fluoride, complete conversion to the pure $\beta$-phase is observed in all cases, even for the highly sodium-deficient $\mathrm{Na}_{5 / 9} \mathrm{YF}_{32 / 9}$ particles. In addition, the resulting particle sizes are slightly affected. Fig. 7 shows that the size of the $\beta$-phase product particles strongly varies with the composition of the $\alpha$-phase particles used for their synthesis (compositions are measured by XRF, see Fig. 4) but also that the presence of additional sodium oleate and $\mathrm{NH}_{4} \mathrm{~F}$ in the solvent results in $\beta$-phase particles which are slightly smaller than those prepared in the absence of sodium oleate and $\mathrm{NH}_{4} \mathrm{~F}$ (see also Fig. S12 and S13†). The latter indicates that the additional sodium and fluoride ions also slightly influence the nucleation of the $\beta$-phase seeds in solution. This weaker effect is similar to the results published by Sui et al. showing that the size of the final $\beta$-phase particles is affected by the amount of metal oleates present during the thermal decomposition of metal trifluoroacetates. ${ }^{31}$

\section{Conclusion}

In conclusion our results show that the size of $\beta-\mathrm{NaYF}_{4}: \mathrm{Yb}, \mathrm{Er}$ upconversion particles prepared in oleic acid/octadecene strongly depends on the composition of intermediately formed $\alpha-\mathrm{NaYF}_{4}: \mathrm{Yb}, \mathrm{Er}$ particles. The ratio of sodium to rare-earth metal in the $\alpha$-phase is one of the key parameters determining the number of $\beta$-phase seeds nucleating during Ostwald-ripening of the $\alpha-\mathrm{NaYF}_{4}: \mathrm{Yb}, \mathrm{Er}$ particles. The size of $\beta-\mathrm{NaYF}_{4}: \mathrm{Yb}, \mathrm{Er}$ upconversion particles can therefore be varied by adjusting this parameter allowing, for instance, to reproducibly prepare phase-pure $\beta-\mathrm{NaYF}_{4}: \mathrm{Yb}$,Er particles in oleic acid/octadecene with a mean diameter smaller than $6 \mathrm{~nm}$.

\section{Acknowledgements}

We thank H. Eickmeier for the TEM investigations.

\section{Notes and references}

1 F. Wang, D. Banerjee, Y. Liu, X. Chen and X. Liu, Analyst, 2010, 135, 1839-1854.
2 H. Wen, H. Zhu, X. Chen, T. F. Hung, B. Wang, G. Zhu, S. F. Yu and F. Wang, Angew. Chem., Int. Ed., 2013, 52, 13419-13423.

3 D. Vennerberg and Z. Lin, Sci. Adv. Mater., 2011, 3, 26-40.

4 L. Wang and Y. Li, Nano Lett., 2006, 6, 1645-1649.

5 S. F. Lim, R. Riehn, W. S. Ryu, N. Khanarian, C.-k. Tung, D. Tank and R. H. Austin, Nano Lett., 2006, 6, 169-174.

6 Z. Li, Y. Zhang and S. Jiang, Adv. Mater., 2008, 20, 47654769.

7 D. K. Chatterjee, A. J. Rufaihah and Y. Zhang, Biomaterials, 2008, 29, 937-943.

8 F. Wang and X. Liu, Chem. Soc. Rev., 2009, 38, 976-989.

9 D. K. Chatterjee, M. K. Gnanasammandhan and Y. Zhang, Small, 2010, 6, 2781-2795.

10 J. Pichaandi, J.-C. Boyer, K. R. Delaney and F. C. J. M. van Veggel, J. Phys. Chem. C, 2011, 115, 19054-19064.

11 P. Zhang, W. Steelant, M. Kumar and M. Scholfield, J. Am. Chem. Soc., 2007, 129, 4526-4527.

12 B. M. van der Ende, L. Aarts and A. Meijerink, Phys. Chem. Chem. Phys., 2009, 11, 11081-11095.

13 A. Shalav, B. S. Richards, T. Trupke, K. W. Kramer and H. U. Gudel, Appl. Phys. Lett., 2005, 86, 013505-013503.

14 E. Downing, L. Hesselink, J. Ralston and R. Macfarlane, Science, 1996, 273, 1185-1189.

15 T. R. Hinklin, S. C. Rand and R. M. Laine, Adv. Mater., 2008, 20, 1270-1273.

16 X. Liu, G. Dong, Y. Qiao and J. Qiu, Appl. Opt., 2008, 47, 6416-6421.

17 F. Wang, Y. Han, C. S. Lim, Y. Lu, J. Wang, J. Xu, H. Chen, C. Zhang, M. Hong and X. Liu, Nature, 2010, 463, 10611065.

18 C. Zhang, H.-P. Zhou, L.-Y. Liao, W. Feng, W. Sun, Z.-X. Li, C.-H. Xu, C.-J. Fang, L.-D. Sun, Y.-W. Zhang and C.-H. Yan, Adv. Mater., 2010, 22, 633-637.

19 S. Baldelli, Nat. Photonics, 2011, 5, 75-76.

20 H. Zhu, X. Chen, L. M. Jin, Q. J. Wang, F. Wang and S. F. Yu, ACS Nano, 2013, 7, 11420-11426.

21 W. J. Kim, M. Nyk and P. N. Prasad, Nanotechnology, 2009, 20, 185301.

22 J. M. Meruga, W. M. Cross, P. S. May, Q. Luu, G. A. Crawford and J. J. Kellar, Nanotechnology, 2012, 23, 395201.

23 T. Blumenthal, J. M. Meruga, P. S. May, J. J. Kellar, W. M. Cross, K. Ankireddy, S. Vunnam and Q. Luu, Nanotechnology, 2012, 23, 185305.

24 J. M. Meruga, A. Baride, W. Cross, J. J. Kellar and P. S. May, J. Mater. Chem. C, 2014, 2, 2221-2227.

25 H.-X. Mai, Y.-W. Zhang, L.-D. Sun and C.-H. Yan, J. Phys. Chem. C, 2007, 111, 13730-13739.

26 J.-C. Boyer, F. Vetrone, L. A. Cuccia and J. A. Capobianco, J. Am. Chem. Soc., 2006, 128, 7444-7445.

27 H.-X. Mai, Y.-W. Zhang, R. Si, Z.-G. Yan, L.-D. Sun, L.-P. You and C.-H. Yan, J. Am. Chem. Soc., 2006, 128, 6426-6436.

28 G. S. Yi and G. M. Chow, Adv. Funct. Mater., 2006, 16, 2324-2329.

29 H.-X. Mai, Y.-W. Zhang, L.-D. Sun and C.-H. Yan, J. Phys. Chem. C, 2007, 111, 13721-13729. 
30 G.-S. Yi and G.-M. Chow, Chem. Mater., 2007, 19, 341-343.

31 Y. Sui, K. Tao, Q. Tian and K. Sun, J. Phys. Chem. C, 2012, 116, 1732-1739.

32 A. D. Ostrowski, E. M. Chan, D. J. Gargas, E. M. Katz, G. Han, P. J. Schuck, D. J. Milliron and B. E. Cohen, ACS Nano, 2012, 6, 2686-2692.

33 H.-S. Qian and Y. Zhang, Langmuir, 2008, 24, 12123-12125.

34 Z. Li and Y. Zhang, Nanotechnology, 2008, 19, 345606.

35 S. Heer, K. Kömpe, H. U. Güdel and M. Haase, Adv. Mater., 2004, 16, 2102-2105.

36 Y. Wei, F. Lu, X. Zhang and D. Chen, Chem. Mater., 2006, 18, 5733-5737.

37 C. Lin, M. T. Berry, R. Anderson, S. Smith and P. S. May, Chem. Mater., 2009, 21, 3406-3413.

38 A. Aebischer, S. Heer, D. Biner, K. Krämer, M. Haase and H. U. Güdel, Chem. Phys. Lett., 2005, 407, 124-128.

39 J. F. Suyver, A. Aebischer, D. Biner, P. Gerner, J. Grimm, S. Heer, K. W. Krämer, C. Reinhard and H. U. Güdel, Opt. Mater., 2005, 27, 1111-1130.

40 J. F. Suyver, J. Grimm, K. W. Krämer and H. U. Güdel, J. Lumin., 2005, 114, 53-59.

41 C. Liu, H. Wang, X. Li and D. Chen, J. Mater. Chem., 2009, 19, 3546-3553.

42 C. Li, Z. Quan, J. Yang, P. Yang and J. Lin, Inorg. Chem., 2007, 46, 6329-6337.

43 C. Li, J. Yang, Z. Quan, P. Yang, D. Kong and J. Lin, Chem. Mater., 2007, 19, 4933-4942.

44 X. Liang, X. Wang, J. Zhuang, Q. Peng and Y. Li, Adv. Funct. Mater., 2007, 17, 2757-2765.

45 J. Zhao, Y. Sun, X. Kong, L. Tian, Y. Wang, L. Tu, J. Zhao and H. Zhang, J. Phys. Chem. B, 2008, 112, 15666-15672.

46 Y. Sun, Y. Chen, L. Tian, Y. Yu, X. Kong, J. Zhao and H. Zhang, Nanotechnology, 2007, 18, 275609.

47 F. Wang and X. Liu, J. Am. Chem. Soc., 2008, 130, 56425643.

48 L. Wang and Y. Li, Chem. Mater., 2007, 19, 727-734.

49 H. Li and L. Wang, Chem. - Asian J., 2014, 9, 153-157.

50 N. J. J. Johnson, W. Oakden, G. J. Stanisz, R. S. Prosser and F. C. J. M. van Veggel, Chem. Mater., 2011, 23, 3714-3722.
51 B. Voß, J. Nordmann, A. Uhl, R. Komban and M. Haase, Nanoscale, 2013, 5, 806-812.

52 D. J. Gargas, E. M. Chan, A. D. Ostrowski, S. Aloni, M. V. P. Altoe, E. S. Barnard, B. Sanii, J. J. Urban, D. J. Milliron, B. E. Cohen and P. J. Schuck, Nat. Nanotechnol., 2014, 9, 300-305.

53 F. Shi and Y. Zhao, J. Mater. Chem. C, 2014, 2, 21982203.

54 A. Podhorodecki, ECS Trans., 2012, 45, 191-198.

55 S. Y. Yu, X. C. Gao, H. Jing, J. Zhao and H. Su, CrystEngComm, 2013, 15, 10100-10106.

56 R. Komban, J. P. Klare, B. Voss, J. Nordmann, H.-J. Steinhoff and M. Haase, Angew. Chem., Int. Ed., 2012, 51, 6506-6510.

57 N. J. J. Johnson, A. Korinek, C. Dong and F. C. J. M. van Veggel, J. Am. Chem. Soc., 2012, 134, 11068-11071.

58 T. Sugimoto, Adv. Colloid Interface Sci., 1987, 28, 65-108.

59 I. M. Lifshitz and V. V. Slyozov, J. Phys. Chem. Solids, 1961, $19,35-50$.

60 C. Wagner, Z. Elektrochem., 1961, 65, 581-591.

61 M. D. Clark, S. K. Kumar, J. S. Owen and E. M. Chan, Nano Lett., 2011, 11, 1976-1980.

62 H.-X. Mai, Y.-W. Zhang, L.-D. Sun and C.-H. Yan, Chem. Mater., 2007, 19, 4514-4522.

63 B. Voss and M. Haase, ACS Nano, 2013, 7, 11242-11254.

64 J. Park, K. An, Y. Hwang, J. Park, H. Noh, J. Kim, J. Park, N. Hwang and T. Hyeon, Nat. Mater., 2004, 3, 891-895.

65 C. A. Schneider, W. S. Rasband and K. W. Eliceiri, Nat. Methods, 2012, 9, 671-675.

66 J. Rodríguez-Carvajal, Physica B, 1993, 192, 55-69.

67 R. E. Thoma, G. M. Herbert, H. Insley and C. F. Weaver, Inorg. Chem., 1963, 2, 1005-1012.

68 P. P. Fedorov, V. B. Aleksandrov, O. S. Bondareva, I. I. Buchinskaya, M. D. Val'kovskii and B. P. Sobolev, Crystallogr. Rep., 2001, 46, 239.

69 N. C. Anderson, M. P. Hendricks, J. J. Choi and J. S. Owen, J. Am. Chem. Soc., 2013, 135, 18536-18548.

70 R. E. Thoma, H. Insley and G. M. Hebert, Inorg. Chem., 1966, 5, 1222-1229. 